\title{
Case Report: The Biomechanical Basis for Rupture of Extensor Mechanism of Knee Joints at Different Sites
}

\author{
Somasekhar Reddy Nallamilli' ${ }^{*}$, (D), Rajyalakshmi Nallamili Reddy ${ }^{1}$ (D), Naveen Chandar Reddy Martha ${ }^{1}$ (D)
}

1. Apollo Hospitals, Jubilee Hills, Hyderabad, India.

\begin{tabular}{|c|c|}
\hline $\begin{array}{l}\text { Use your device to scan } \\
\text { and read the article online }\end{array}$ & $\begin{array}{l}\text { ditation Reddy Nallamilli S, Reddy RN, Reddy Martha NC, Reddy Nallamilli S. The Biomechanical Basis for Rupture of } \\
\text { Extensor Mechanism of Knee Joints at Different Sites. Journal of Research in Orthopedic Science. 2021; 8(1):51-56. http:/ } \\
\text { dx.doi.org/10.32598/JROSJ.8.1.745.1 } \\
\text { doi: } \text { : http://dx.doi.org/10.32598/JROSJ.8.1.745.1 }\end{array}$ \\
\hline
\end{tabular}

\section{(c) (1) (8)}

Article info:

Received: 05 Nov 2020

Revised: 25 Nov 2020

Accepted: 27 Dec 2020

Available Online: 01 February 2021

Keywords:

Quadriceps tendon tear, Ligamentum patella tear, Bilateral knee extensor mechanism injury, Quadriceps tendon repair, Patellar tendon repair and augmentation

\begin{abstract}
A B S T RA C T
Rupture of the extensor mechanism of knee joints is rare in patients without any systemic disease. Many reports have described tendons getting ruptured at various sites of the extensor mechanism. We report a case of patellar tendon avulsion from the tibial tuberosity on the right side and quadriceps tendon avulsion from the patella on the left side sustained in one accident. A 54 years old man presented with the above non-identical bilateral extensor mechanism rupture who had no pre-existing systematic diseases and was not on any steroid treatment. Tendon ruptures were always related to systemic degenerative/inflammatory conditions or usage of steroids. However, the reason for the rupture of different parts of the extensor mechanism after minor trauma in case of tendinopathy or after a significant trauma in a normal patient is not well explained. We believe that the degree of knee flexion at the time of injury plays an important role in the site of rupture of the extensor mechanism.
\end{abstract}

\section{Introduction}

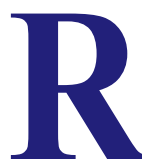

upture of the extensor mechanism of knee joints is rare and even rarer in patients without any systemic disease. Bilateral rupture is also even uncommon. Kellersmann [1] and Lu et al. [2] reported cases with bilateral extensor mechanism disruption at the same level on both sides without any preexisting causes and Yasen et al. [3] reported a case of non-identical bilateral rupture of extensor mechanism in a patient with hyperlipidemia $[4,5]$. Most studies have explained tendon ruptures either unilateral or bilateral, based on the underlying tendinopathy irrespective of the site of disruption. We aimed at explaining the non-identical rupture of the extensor mechanism in the following case on a biomechanical basis.

\section{Case Report}

A 54 years old man presented with a history of pain in both of his knee joints with swelling, inability to stand or

* Corresponding Author:

Somasekhar Reddy Nallamilli, MD.

Address: Apollo Hospitals, Jubilee Hills, Hyderabad, India.

Phone: +91 (984) 8025355

E-mail: nreddy12@yahoo.com 
walk following an injury sustained while trekking in snow ten days ago. His right leg got stuck in the snow and he tried to pull it out of the snow forcing his left foot heavily onto the snow, while fellow men were trying to pull him up. He felt a sudden pain in both knees, which buckled on attempting to stand. He was unable to weight bear. The swelling was developed in both knees. He denied any prior trauma or any pre-existing symptoms in either of the knees. He had no known medical problems and was not a smoker or an alcoholic person. He had not taken steroids in the past. At the presentation, he was fit and healthy.

The patient had swelling in both knees with bruising but without any skin wounds. A depression was felt below the patella on the right side with no palpable patellar tendon. A wide defect of about one inch was palpable in the quadriceps just above the patella on the left knee (Figure 1). He was unable to extend either of the knees or hold either the knees in extension when passively positioned. Plain radiographs of knees revealed high-riding patella on the right side with normally located patella on the left side (Figure 2). Magnetic Resonance Imaging (MRI) confirmed rupture of the patellar tendon on the right and quadriceps tendon on the left (Figure 3). There were no other intraarticular abnormalities.

During surgery, patellar tendon avulsion from the tibial tuberosity was confirmed on the right side. This was sutured to the soft tissue around the tibial tuberosity with $1 / 0$ vicryl and an anchor to the tuberosity. This repair was protected with a cerclage wire loop passing through drill holes at the middle of the patella and the tibial tuberosity. On the left side, the quadriceps tendon was found to be avulsed from the superior pole of the patella with disruption of the medial and lateral patellar retinacula. However, the torn tendon appeared healthy with no obvious degenerative changes in tendon. A subsequent bi- opsy report of the tendon revealed normal architecture. A Bunnel's pull-out stitch with Ethibond -No [5]. through the quadriceps tendon was taken and was secured to the superior pole of the patella. The rents in the medial and lateral retinacula were repaired using absorbable suture. Wounds of both knee joints healed well (Figure 4).

Post-operatively, he had supervised rehabilitation for 12 weeks followed by a home exercise program. He regained 5/5-muscle power (MRC scale) of the quadriceps by 6 months on both sides with a full range of knee movements (Figure 5). X-rays confirmed anatomically positioned patellae on both sides with broken cerclage wire on the right side, which was asymptomatic (Figure 6). At four and six years following the injury, there was no weakness of knee extension and there was no evidence of tendinopathy at the knee or other parts of the body.

\section{Discussion}

Patellar tendon rupture is commonly associated with systemic diseases, such as rheumatoid arthritis, lupus erythematosus, and hyperparathyroidism. Repetitive microtrauma and long-term corticosteroid use are also known to precipitate $[4,5]$. Bilateral tendon ruptures are not very common, with only about 50 reported cases in the English and German literature [4]. Patellar tendon rupture is the third most common cause of extensor dysfunction, after patellar fracture and quadriceps tendon rupture [6]. The extensor mechanism is at risk of rupture during forced knee flexion against a strongly contracting quadriceps. The 'give way' point could be in the substance of the quadriceps tendon, its attachment to the patella, mid-patella (fracture of patella), the inferior pole of the patella at the attachment of the patellar tendon, and the mid-substance of the tendon or its attachment to the tibial tuberosity. Zernike et al. [7] reported that a force

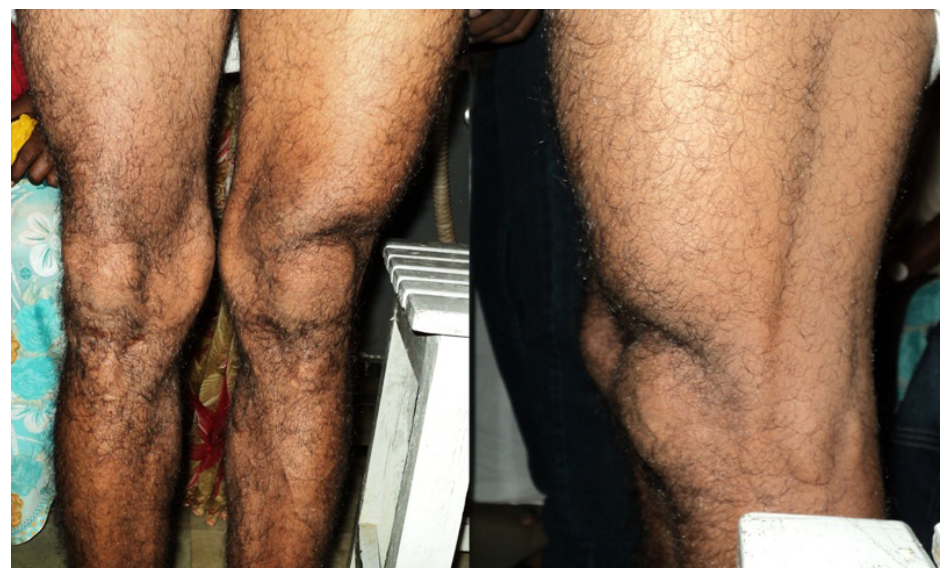

Figure 1. Preoperative clinical pictures showing normal-looking right knee and depression above the patella on the left side 


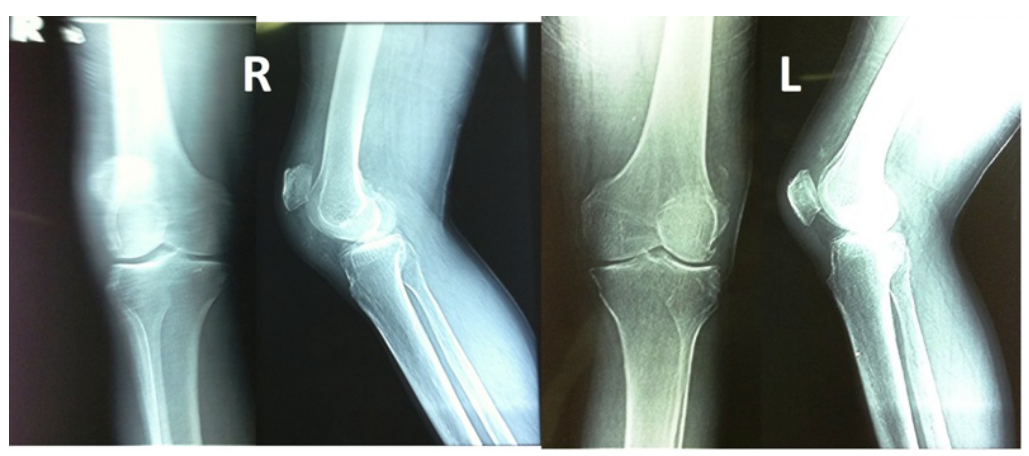

Orthopedic Science

Figure 2. Preoperative x-rays of the knee joints showing proximally migrated patella on the right side and relatively normally placed patella in the left knee

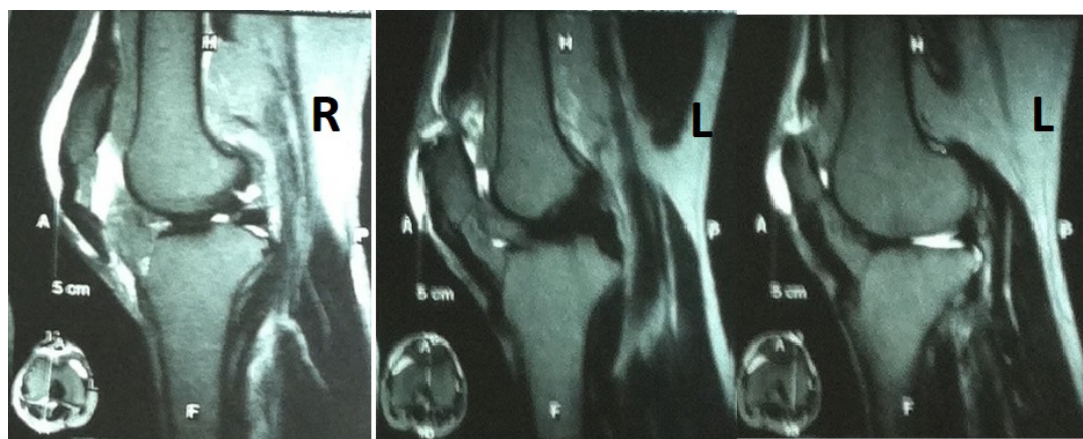

Orthopedic Science

Figure 3. Preoperative Magnetic Resonance Imaging (MRI) images of the knee joints: Right knee showing avulsion of ligamentum patella from the tibial tuberosity and left knee showing rupture of quadriceps from the superior pole of the patella

of 17.5 times the body weight is required to rupture the patella tendon [7]. Men are more prone to bilateral patellar tendon rupture compared with women [5:1 ratio] [8]. Siwek et al. [9] found that $28 \%$ of the reported bilateral tendon ruptures were misdiagnosed on initial examina- tion [9]. Pathophysiology of tendon ruptures in cases of systemic diseases is thought to be due to inflammatory changes and amyloid deposition that alter the structure of the tendon [10]. Injection of steroids locally into the tendon may affect collagen synthesis and compromise

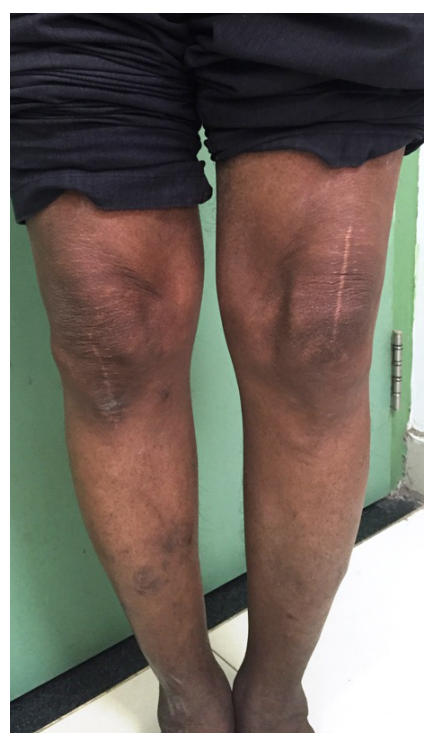

Orthopedic Science

Figure 4. Postoperative clinical picture showing well-healed surgical scars below the patella on the right side and above the patella on the left side 


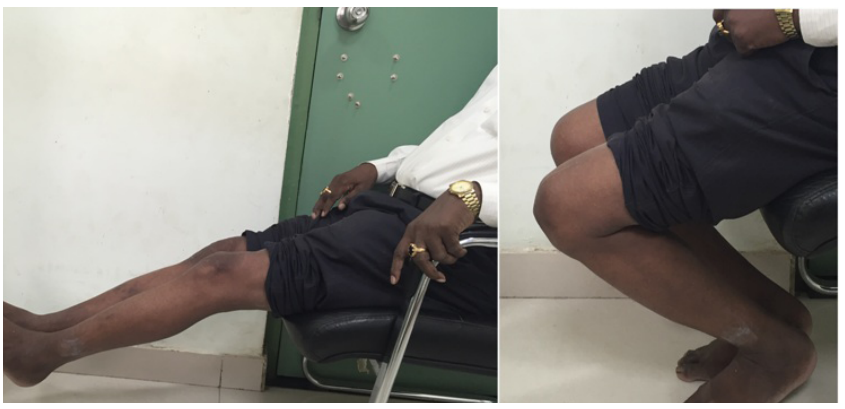

Figure 5. Clinical pictures at 2 years follow-up showing the range of movements of both knee joints

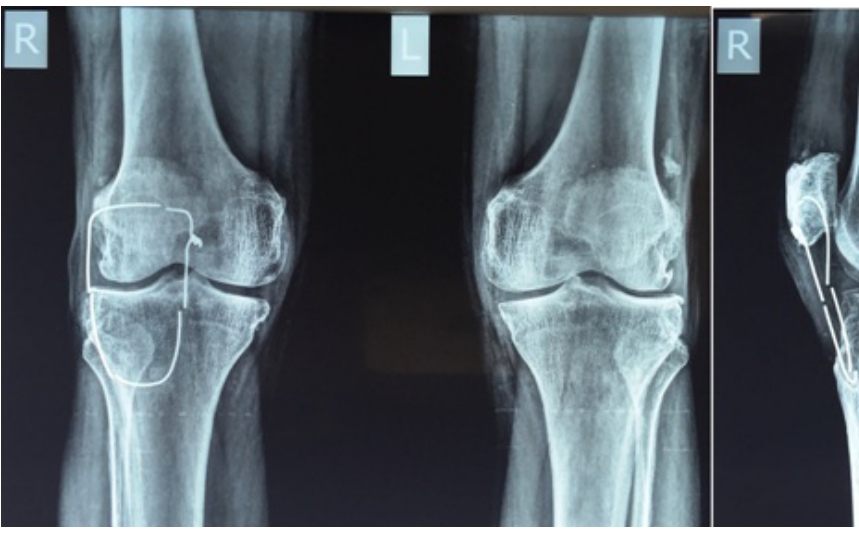

Figure 6. X-rays of both knee joints at 2 years follow-up

blood supply, thereby weakening the tendon $[4,5,11]$. Bagherifard et al. reported simultaneous bilateral quad-

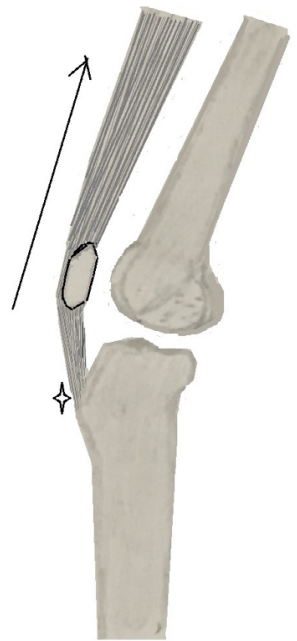

Orthopedic Science

Figure 7. Diagrammatic representation of quadriceps contraction with the knee in $<15^{\circ}$ of flexion

The resultant linear force gets transmitted directly to the tibial tuberosity (point of tear is marked)

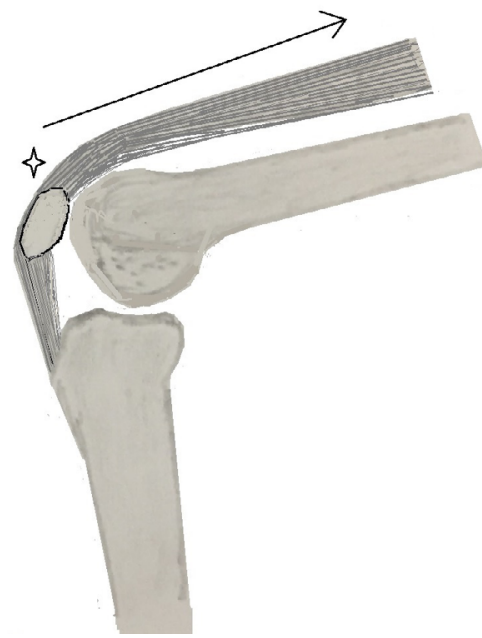

Orthopedic Science

Figure 8. Diagrammatic representation of quadriceps contraction with the knee in $>90^{\circ}$ of flexion

The resultant force is concentrated over the superior pole of the patella, which is firmly locked in the trochlea (point of tear is marked) 
ing a minor trauma who consumed anabolic androgenic steroids [12].

Chronic inflammation generated by repetitive microtrauma may predispose to degeneration of the tendon by interfering with the healing process and is referred to as Davidson's theory in the literature $[13,14]$. The patient in this report had patellar tendon avulsion from the tibial tuberosity on the right side and quadriceps tendon rupture from the superior pole of the patella on the left side. This variance in tear pattern from one knee to another has only been reported in two other cases in the literature and in a case of hyperlipidemia $[3,13,15]$. It is interesting to note that his right leg got stuck in the snow and it is likely that this knee was relatively straight at the time of injury. He pushed the snow with his left foot and most probably, the left knee is in flexion while trying to lift him off the snow.

Twice as much torque is required to extend the knee in the final $15^{\circ}$ as is necessary to bring it from a fully flexed position to $15^{\circ}$ [16] (Figure 7). Much of the central part of the tendon of the quadriceps gets inserted into the superior pole of the patella over a 'large cross-sectional area'. However, a significant portion of the tendon continues over the anterior surface of the patella and as medial and lateral patellar retinaculum directly into the ligamentum patella. This patellar tendon gets inserted into the tibial tuberosity, which, however, has a very small cross-sectional area'. Thus, the force generated by the vigorous contraction of the quadriceps with the knee in a relatively extended position gets transmitted directly to the point of insertion at the tibial tuberosity, which is the weakest link in this position. Hence, the ligamentum patella gets avulsed. There would be no stress riser at the insertion of quadriceps at the superior pole of the patella as patella and its retinaculum move proximally as one unit with quadriceps contraction. Whatever is the mechanism of injury or the underlying pathology the treatment of choice in most of the cases is surgical repair [10]

The patella-femoral contact zones are dynamic and shift with varying degrees of knee flexion. The patella engages the trochlear sulcus at approximately $20 \mathrm{D}$ of flexion. With increasing knee flexion, a horizontal band of contact area across the patellar facets moves proximally and reaches a maximum at $90 \mathrm{D}$ of flexion. Corresponding with the proximal shift of contact on the patella, the contact zone on the femur shifts distally on the trochlea $[17,18]$.

Midway between $20 \mathrm{D}$ and $90 \mathrm{D}$ of the lower half of the patella is engaged in the trochlea leaving the upper half still not supported by the trochlea. In this position, powerful contraction of the quadriceps produces a tensile force on the patella leading to a transverse fracture. However, with the knee in $>90^{\circ}$ flexion and patella firmly locked in the trochlear groove, the same quadriceps contraction force gets concentrated over the superior pole of an immobile patella and the quadriceps tendon gets avulsed (Figure 8). In a flexed position, the superficial fibers of the quadriceps tendon at the superior pole of the patella are under tension and are the first to break as they may have reached the limit of their stretch. With continued force, the tear extends to deep fibers and subsequently on to medial and lateral retinaculum.

Disruption of the extensor mechanism may occur after minor trauma in cases with systemic inflammatory conditions or local tendinopathy and a healthy tendon may tear due to a severe force. Local steroid infiltrations can lead to tears in the same place due to direct damage to the tendon [19]. It appears that the force of muscle contraction or the systemic pathology may not play any crucial role in deciding the site of tendon disruption. The degree of knee flexion at the time of injury may play a very significant role in the site of disruption of the extensor mechanism.

There are very few recent biomechanical studies on extensor mechanism injuries. Any future reports of similar cases, if they include the position of the knee at the time of injury may throw further light on the mechanism of these injuries concerning the site of ruptures.

\section{Ethical Considerations}

\section{Compliance with ethical guidelines}

All ethical principles are considered in this article. The participants were informed of the purpose of the research and its implementation stages.

\section{Funding}

This research did not receive any grant from funding agencies in the public, commercial, or non-profit sectors.

\section{Authors' contributions}

All authors equally contributed to preparing this article.

\section{Conflict of interest}

The authors declared no conflict of interest. 


\section{Acknowledgments}

This paper was prepared from a case treated by us at our institution. The material was not published in any form, neither as a thesis nor as a podium presentation by any of the authors in the past

\section{References}

[1] Kellersmann R, Blattert TR, Weckbach A. Bilateral patellar tendon rupture without predisposing systemic disease or steroid use: A case report and review of the literature. Arch Orthop Trauma Surg. 2005; 125(2):127-33. [DOI:10.1007/ s00402-004-0782-2] [PMID]

[2] Lu HD, Cai DZ, Wang K, Zeng C. Simultaneous bilateral patellar tendon rupture without predisposing systemic disease or steroid use: A case report. Chin J Traumatol. 2012; 15(1):54-8. [PMID]

[3] Yasen SK, Foster AJ, Thakrar RR. Non-identical bilateral rupture of the extensor mechanism of the knee in a patient with hyperlipidemia: A case study. J Orthop Case Rep. 2019; 9(4):88-91. [PMID] [PMCID]

[4] Clark SC, Jones MW, Choudhury RR, Smith E. Bilateral patellar tendon rupture secondary to repeated local steroid injections. J Accid Emerg Med. 1995; 12(4):300-1. [DOI:10.1136/ emj.12.4.300] [PMID] [PMCID]

[5] Morgan J, McCarty DJ. Tendon ruptures in patients with systemic lupus erythematosus treated with corticosteroids. Arthritis Rheum. 1974; 17(6):1033-6. [DOI:10.1002/ art.1780170616] [PMID]

[6] Rose PS, Frassica FJ. Atraumatic bilateral patellar tendon rupture, A case report and review of the literature. J Bone Joint Surg Am. 2001; 83(9):1382-6. [DOI:10.2106/00004623200109000-00014] [PMID]

[7] Zernicke RF, Garhammer J, Jobe FW. Human patellartendon rupture. J Bone Joint Surg Am. 1977; 59(2):179-83. [DOI:10.2106/00004623-197759020-00007] [PMID]

[8] Taylor BC, Tancev A, Fowler T. Bilateral patellar tendon rupture at different sites without predisposing systemic disease or steroid use. Iowa Orthop J. 2009; 29:100-4. [PMID] [PMCID]

[9] Siwek CW, Rao JP. Ruptures of the extensor mechanism of the knee joint. J Bone Joint Surg Am. 1981; 63(6):932-7. [DOI:10.2106/00004623-198163060-00010] [PMID]

[10] Kannus P, Józsa L. Histopathological changes preceding spontaneous rupture of a tendon. A controlled study of 891 patients. J Bone Joint Surg Am. 1991; 73(10):1507-25. [DOI:10.2106/00004623-199173100-00009] [PMID]

[11] Van Glabbeek F, De Groof E, Boghemans J. Bilateral patellar tendon rupture: Case report and literature review. J Trauma. 1992; 33(5):790-2. [DOI:10.1097/00005373-19921100000035] [PMID]

[12] Bagherifard A, Jabalameli M, Rezazadeh J, Ghaffari S, Tabrizian P. Simultaneous bilateral quadriceps tendon rupture follow- ing a low-energy trauma in a male body builder with the history of anabolic-androgenic steroids consumption. J Res Orthop Sci. 2018; 5(2):1-4. http:/ /jros.iums.ac.ir/article-1-233-en.html

[13] Peters KM, Bücheler D, Westerdorf G. Bilateral rupture of the patellar ligament in diabetes mellitus. Unfallchirurg. 2000; 103(2):164-7. [DOI:10.1007/s001130050029] [PMID]

[14] Splain SH, Ferenz C. Bilateral simultaneous infrapatellar tendon rupture: support for Davidsson's theory. Orthop Rev. 1988; 17(8):802-5. [PMID]

[15] Sochart DH, Shravat BP. Bilateral patellar tendon disruption-a professional predisposition? J Accid Emerg Med. 1994; 11 (4):255-6. [DOI:10.1136/emj.11.4.255] [PMID] [PMCID]

[16] Lieb FJ, Perry J. Quadriceps function. An anatomical and mechanical study using amputated limbs. J Bone Joint Surg Am. 1968; 50 (8):1535-48. [DOI:10.2106/00004623196850080-00003] [PMID]

[17] Aglietti P, Insall JN, Walker PS, et al. A new patellar prosthesis. Design and application. Clin Orthop Relat Res. 1975; (107):17587. [DOI:10.1097/00003086-197503000-00023] [PMID]

[18] Goodfellow J, Hungerford DS, Zindel M. Patello-femoral joint mechanics and pathology. 1. Functional anatomy of the patello-femoral joint. J Bone Joint Surg Br. 1976; 58(3):287-90. [DOI:10.1302/0301-620X.58B3.956243] [PMID]

[19] Jabalameli M, Bagherifard A, Hadi H, Yoosefzadeh A, Yahyazadeh $\mathrm{H}$, Rezazadeh J, et al. Long-term outcome after surgical treatment of acute and chronic quadriceps tendon rupture. J Res Orthop Sci. 2018; 5(1):e63127. http://jros. iums.ac.ir/article-1-226-en.html 